\title{
La medicina basada en evidencia rompe paradigmas, un aprendizaje de la pandemia
}

\author{
DOI 10.5377/alerta.v4i3.10968 \\ David Alberto Santos Hernández \\ Universidad de El Salvador, San Salvador, El Salvador. \\ Correspondencia \\ 曰dsantos2894@gmail.com \\ (D) 0000-0002-6099-0195
}

\section{Sra. Editora.}

La medicina basada en evidencia (MBE) se refiere a la integración de la mejor evidencia disponible con la experiencia clínica y valores de los pacientes'. Su introducción a la práctica clínica permite evaluar correctamente los estudios clínicos (métodos y resultados estadísticos) para utilizar las terapias que produzcan mayor seguridad con un costo-beneficio asequible, considerándose los aspectos éticos de las intervenciones. Su aplicación trasciende al ámbito clínico, contribuyendo a generar un pensamiento crítico. Sus principios y métodos pueden ser extrapolados a la gestión en salud bajo el término "gestión basada en la evidencia», lo que requiere el análisis de la información para aplicarla en el ámbito público y evaluar sus resultados para realizar modificaciones y mejorar su impacto en la población. Este proceso permite priorizar recursos, siempre limitados, a estrategias seguras y efectivas para cubrir necesidades siempre ilimitadas.

Durante la pandemia de la COVID-19, la necesidad y deficiencia de la incorporación de la MBE en diversas áreas de la salud ha sido más evidente a raíz de la cantidad y calidad de información generada durante esta crisis, la cual ha tenido repercusiones importantes sobre pacientes individuales, grupos de pacientes (implementación de protocolos de atención) y sobre las sociedades (orientación de recursos económicos para la implementación de esos protocolos). Muchas de las intervenciones prometedoras y protocolos de atención se basaron en informes de calidad cuestionable, siendo la hidroxicloroquina uno de los ejemplos más emblemáticos ${ }^{2}$, que posteriormente el estudio RECOVERY demostró la ausencia de cualquier beneficio que se le pudo atribuir, al igual que sucedió con la aspirina, azitromicina, colchicina, lopinavir-ritonavir y recientemente con la ivermectina ${ }^{3}$.

Concuerdo con la doctora Sandoval ${ }^{4}$, al exponer que es necesario mantenerse informados para atender los problemas de salud de las personas y que la pandemia de la COVID-19 ha demostrado que los sistemas de comunicación científica no satisfacen las necesidades de la ciencia y la sociedad. Sin embargo, este aprendizaje en realidad es una de las caras de una moneda. La primera cara es la necesidad de oro: información. La segunda es: «no todo lo que brilla es oro» (se debe analizar la evidencia) y esta última es la que más ha marcado la pandemia.

Los paradigmas en salud han evolucionado desde una concepción mágico-religiosa hasta a un sistema científico. Previo a la implementación de las estrategias de la $M B E$, el ejercicio médico se practicaba bajo el manto de las llamadas ciencias empíricas $^{5}$ y las intervenciones médicas que durante este tiempo se realizaron, se transmitían de profesor a estudiante en forma de dogma, sin ser cuestionados; guiados por el sentido común. Estas intervenciones se volvieron costumbres y finalmente leyes. Una vez que adquirieron esta categoría, transcurrieron varios siglos antes que surgiera el primer concepto de MBE cuya noción principal se puede resumir con la frase: «Nullius in verba» (en la boca de nadie); es decir, no
6

\section{ACCESO ABIERTO}

Evidence based medicine breaks paradigms, a learning from the pandemic

\section{Citación recomendada:}

Santos Hernández DA. La medicina basada en evidencia rompe paradigmas, un aprendizaje de la pandemia. Alerta. 2021; 4(3):181-182. DOI: 10.5377/alerta.v4i3.10968

\section{Recibido:}

2 de marzo de 2021

\section{Aceptado:}

9 de julio de 2021

\section{Publicado:}

26 de julio de 2021

\section{Contribución de autoría:} Elaboración total del autor.

\section{Conflicto de intereses:}

Autor declara no tener ningún tipo de conflicto de interés. 
influenciada por ninguna persona (independientemente de su renombre, posición social o laboral) y que las decisiones se fundamentaran sobre una base científica. Estas premisas ciertamente iban en contra de un paradigma con años de fundamento.

Actualmente se pueden observar ciertos remanentes del paradigma mágicoreligioso; el que concebía la enfermedad como hechos sobrenaturales que se debían aceptar, porque así eran. De forma similar se transmitió el conocimiento médico y ahora sucede con la interpretación de la información científica.

Lograr que los usuarios de las publicaciones médicas, profesionales y gerentes de salud, logren diferenciar la información científica de aquella que tiene la mayor evidencia, es el reto actual de la MBE, y el que ha sido más patente durante la pandemia. Dejar atrás los remanentes del paradigma mágico-religioso, que requieren la atención e involucramiento de todos los actores clave: investigadores, docentes, médicos, asociaciones médicas, asociaciones estudiantiles, etc. Aunque ya se han realizado avances, se espera una resistencia cultural anticipada según palabras de Martínez-Lovo: «siempre fue más fácil explicar los fenómenos naturales bajo conceptos aportados por el sentido común, sin jamás detenerse a reflexionar el porqué de tales eventos desde una perspectiva científica» ${ }^{6}$.

Para abordar los problemas de las dos caras de la moneda (necesidad de información y forma de análisis de la información) y lograr el cambio de paradigma, se han realizado contribuciones significativas: la revista Alerta abre un importante canal de comunicación científica en español, de acceso abierto, lo que hace accesible la información al disminuir las barreras económicas y del lenguaje. Representa un estímulo para que actores nacionales interesados en producir científicamente puedan acceder al mundo científico. Por otro lado, recursos como «El $A B C$ de la lectura crítica», permiten conocer las nociones elementales para el análisis de la información científica. De igual forma, son destacables los aportes de estudiantes del área de la salud en espacios como Students for Best Evidence, sitio que recientemente ha sido traducido al español y portugués, permitiendo disminuir la barrera del lenguaje y que abre espacios para la producción científica estudiantil de forma gratuita, a la vez que instruyen sobre conceptos relacionados con MBE y análisis de publicaciones científicas, demostrando que todos los actores clave pueden agregar su grano de arena.

La emergencia mundial actual ha mostrado la evidencia más notoria de lo diná- mica que es la medicina y que la valoración adecuada de la información científica depende de acentuar el cambio de paradigma con implementación de la MBE. Los profesionales que se atrevan a embarcarse en ese mar profundo se adaptarán de mejor manera a los cambios que ya se están generando y, consecuentemente, tendrán mayores posibilidades de éxito. Es necesario recordar que el éxito en las ciencias médicas se resume en vidas humanas. Estas consideraciones sobre la MBE y sus beneficios sobre los pacientes hacen que su aplicación también sea un tema ético para los profesionales de la salud.

Avanzar en la transición de paradigma no es un camino fácil y se tiene la oportunidad de ser emprendedores en nuestro campo. Sueño con ver ese cambio cultural aplicándose en todos los niveles.

\section{Referencias bibliográficas}

1. Haynes RB, Devereaux PJ, Guyatt GH. Clinical expertise in the era of evidence-based medicine and patient choice. Vox Sang. 2002;83(s1):383-386. DOI: 10.1111/j.14230410.2002.tb05339.x

2. Aronson JK, DeVito N, Ferner RE, Mahtani KR, Nunan D, Plüddemann A. The ethics of COVID-19 treatment studies: too many are open, too few are double-masked. Centre for Evidence-Based Medicine. 2020 Fecha de consulta: 28 de mayo de 2021. Disponible en: https://www.cebm.net/ covid-19/the-ethics-of-covid-19-treatmentstudies-too-many-are-open-too-few-aredouble-masked/

3. Mullard A. RECOVERY 1 year on: a rare success in the COVID-19 clinical trial landscape. Nat Rev Drug Discov. 2021;20(5):336-337. DOI: 10.1038/d41573021-00068-w

4. Sandoval López X. La revista Alerta y su aporte a la comunicación científica. Alerta. 2021;4(1):3-4 DOI: 10.5377/alerta.v4i1.10761.

5. Huicho L, Yhuri Carreazo N, Gonzales C. La Medicina Basada en la Evidencia: ¿mejoró la medicina que practicamos y enseñamos? An Fac med. 2013;74(3):231-5. DOI: 10.1538

6. Martínez-Lovo FJ. La cultura sin ciencia: irracionalismo cultural. Alerta. 2020;3(2):135136. DOI: 10.5377/alerta.v3i2.97431/anales. v74i3.2641.

7. Rodríguez Funes MV. El ABC de la lectura crítica científica para estudiantes y profesionales de la salud: nociones elementales para principiantes. 1ra Edición. San Salvador. Editorial Universitaria (UES); 2018. $96 \mathrm{p}$ 